\title{
Auf Wiedersehen
}

\section{Matthias Scholer}

Chefredaktor SÄZ

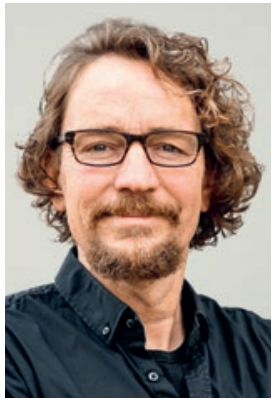

Dies ist das letzte $\mathrm{Zu}$ guter Letzt. Einerseits für dieses Jahr, andererseits für mich als Chefredaktor der Schweizerischen Ärztezeitung (SÄZ). Doch der Reihe nach.

Traditionsgemäss schaut der Chefredaktor (ja, es waren bislang nur Männer) jeweils Ende Jahr auf die vergangenen zwölf Monate zurück. Daran wollen wir auch in diesen unruhigen Zeiten nichts ändern. 2021 war für uns neben dem alles dominierenden Thema der Pandemie auch ein Jahr der personellen Wechsel. Die grösste Veränderung bei der SÄZ hängt direkt mit dem Wechsel an der Führungsspitze der FMH zusammen. Yvonne Gilli wurde Ende Oktober 2020 zur neuen FMH-Präsidentin gewählt. Mit der Übernahme der Amtsgeschäfte am 1. Februar 2021 löste Yvonne Gilli den scheidenden Präsidenten Jürg Schlup als Mitglied der Redaktionskommission ab. Jürg Schlups regelmässige Teilnahme an den Redaktionssitzungen zeigte uns, dass ihm das "gelbe Heft» am Herzen lag. Erfreulicherweise stand von Beginn weg fest, dass sich die neue FMH-Präsidentin ebenfalls als Redaktionsmitglied aktiv bei der Weiterentwicklung der SÄZ einbringen möchte. Seither ist die FMH mit zwei Frauen in der Redaktionskommission vertreten: mit Yvonne Gilli und Charlotte Schweizer, der Leiterin der Kommunikation. Ich möchte mich an dieser Stelle herzlich bei Jürg Schlup, Yvonne Gilli und Charlotte Schweizer, aber auch dem ganzen Team der FMH für das Engagement rund um die SÄZ und die stets gute Zusammenarbeit bedanken.

Das Jahr 2021 war für uns neben dem alles dominierenden Thema der Pandemie auch ein Jahr der personellen Wechsel.

Doch es gab noch mehr personelle Wechsel bei der SÄZ. Seit diesem April verstärkt Eva Mell unser Redaktions- und Produktionsteam in Muttenz. Beim Schweizerischen Ärzteverlag EMH ist die versierte Wissenschaftsjournalistin einerseits als Managing Editor für die fristgerechte Produktion der FMH-Artikel und die Artikeldisposition der einzelnen Ausgaben zuständig. Andererseits verfasst Eva Mell regelmässig Artikel für unsere Zeitschrift. Im Sommer stiess zudem Rahel Gutmann als Junior-Redaktorin zu uns. Die Historikerin gibt den zahlreichen Artikeln den letzten sprachlichen Schliff und begleitet diese durch die Produktion bis zur Druckfreigabe.
Neben dem Willkommenheissen der neuen Personen hiess es aber auch Abschied nehmen. Im Oktober trat Annette Eichholtz ihren wohlverdienten Ruhestand an. Viele unserer Leserinnen und Leser hatten in der Vergangenheit mit Annette Eichholtz bereits Kontakt. Kein Wunder, schliesslich arbeitete sie während zwölf Jahren in verschiedenen Positionen für die SÄZ.

\section{Ich werde der Lancierung einer überarbeiteten} SÄZ entgegenfiebern. Schliesslich trage ich nach so langer Zeit das SÄZ-Gen in mir.

Zudem werde ich auf Ende Januar EMH verlassen. Nach zehn Jahren, während denen ich in verschiedenen Funktionen für das Verlagshaus tätig sein durfte, habe ich mich entschieden, bei einem anderen Medizinverlag eine neue Herausforderung anzunehmen. Ich werde jedoch auch in dieser neuen Position der Kommunikation standes- und gesundheitspolitischer Themen treu bleiben.

Und wie geht es nun für die SÄZ weiter? Obwohl wir die Zeitschrift trotz der noch immer spürbaren, Covidbedingten Einschränkungen relativ gut durch das vergangene Jahr manövriert haben, bereiten uns die in der gesamten Medienwelt rückläufigen Werbeeinnahmen Sorgen. Nach einem Beschluss der Ärztekammer werden die SÄZ und das Swiss Medical Forum seit 2019 vollumfänglich durch Werbeeinnahmen finanziert. Auf diese Abhängigkeit vom Inseratemarkt musste der EMH-Verlag mit verschiedenen Massnahmen reagieren, um weiterhin unsere Leserschaft mit qualitativ hochstehenden Inhalten bedienen zu können. Einer dieser Handlungsstränge betrifft die Überarbeitung des Inhaltskonzeptes der SÄZ. Ich durfte bei der Strategieentwicklung im laufenden Jahr mitwirken die Keimzelle für ein den künftigen Herausforderungen gerecht werdendes Zeitschriftenkonzept ist gelegt. Auch wenn ich nun das Team verlasse und nicht mehr als «Geburtshelfer» zur Verfügung stehe, werde ich der Lancierung einer überarbeiteten SÄZ entgegenfiebern, schliesslich trage ich nach so langer Zeit das SÄZ-Gen fest in mir.

Ich danke allen Beteiligten für eine spannende, erlebnis- und lehrreiche Zeit und wünsche Ihnen, liebe Leserinnen und Leser, weiterhin gute Unterhaltung bei der Lektüre und einen optimalen Start ins neue Jahr. 